\title{
Targeting bacterial virulence
}

\section{By Brian Moy, Staff Writer}

Bacterial virulence factors are attractive therapeutic targets because they do not affect bacterial growth and thus should be under less selective pressure to develop drug resistance. One drawback, however, is that virulence factors are often species-specific and thus unlikely to be useful as broad-spectrum targets.

Researchers at the University of Texas Southwestern Medical Center now have shown that the virulence target QseC is conserved across multiple types of bacteria, and they have identified a QseC inhibitor that could offer broad-spectrum antibacterial efficacy. ${ }^{1}$

QseC is a membrane-embedded histidine kinase that bacteria use to recognize the host signaling molecules epinephrine and norepinephrine, and bacterial autoinducer-3 (AI-3), a compound involved in bacterial cell-to-cell signaling. Once one of these molecules has

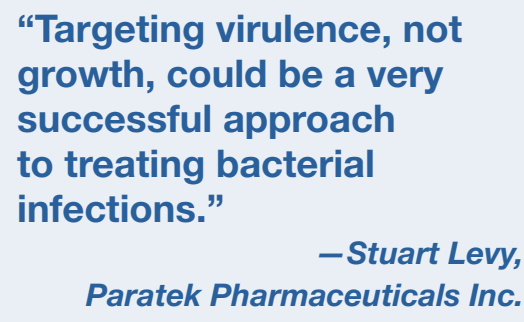

\section{Resisting the resistance}

Michael Pucci, senior director of antimicrobial drug discovery at Achillion Pharmaceuticals Inc., said the paper demonstrates a "novel approach to fighting bacterial infections by which you can shut off certain genes that encode virulence proteins or toxins that enable pathogens to cause disease, without necessarily preventing the bacteria from colonizing or invading its host."

Achillion's elvucitabine, a nucleoside analog viral polymerase inhibitor, has completed Phase II testing to treat HIV. The company is also developing ACH-702, an inhibitor of the DNA replication enzymes gyrase, topoisomerase IV and primase. It is in preclinical testing for use as a broad-spectrum antibiotic.

Stuart Levy, CSO at Paratek Pharmaceuticals Inc., professor in the departments of molecular biology and microbiology and medicine at Tufts University and director of Tufts' been detected, QseC triggers a complex signaling cascade that activates, among other targets, bacterial virulence genes. $^{2}$

Blocking QseC signaling could prevent a bacterial pathogen from identifying host signaling molecules and thus inhibit the bacteria from recognizing the host environment and becoming virulent.

According to the authors of the Science paper, "because QseC is present in many important animal and plant pathogens, drugs that target this sensor kinase have the potential to have broad spectrum."

Using a high throughput screen, the UT Southwestern researchers identified LED209, a substituted benzenesulfonamide that inhibits QseC-dependent virulence-gene activation. In cultures of Escherichia coli, Salmonella typhimurium and Francisella tularensis, the small molecule lowered expression of several virulence genes.

In mice infected with S. typhimurium, 80\% of those given LED209 were alive 24 hours after infection compared with $30 \%$ of untreated controls. Additionally, in mice infected with F. tularensis, $80 \%$ of treated mice were alive 9 days after infection compared with $10 \%$ of untreated controls.

Ongoing SAR studies of LED209 are aimed at developing a nonsystemically absorbed form of the molecule to increase its antibacterial efficacy in the gut.

Vanessa Sperandio, the principal investigator and corresponding author on the Science paper, told SciBX that "by blocking QseC
Center for Adaptation Genetics and Drug Resistance, told SciBX he believes that "targeting virulence, not growth, could be a very successful approach to treating bacterial infections."

Paratek is developing multiple adaptational response (MAR) inhibitors as broad-spectrum agents that target bacterial virulence. According to the company, the mar operon is a set of genes present in most, if not all, enteric Gram-negative bacteria that confers survival traits to the bacterium. Thus, Levy said that "MAR inhibitors could block the expression of many virulence genes at a single time."

Paratek's lead MAR inhibitors are in preclinical testing to treat nosocomial respiratory and urinary tract infections.

Neither MAR inhibitors nor LED209 kill bacteria or inhibit its growth. Although that sounds like a drawback, Sonia Escaich, VP and CSO at Mutabilis S.A., thinks it gives antivirulence compounds an advantage over classic antibiotics.

"Rather than targeting bacterial genes that are essential for basic metabolism in vitro, the virulence targets are essential functions for host-pathogen interactions, which still allow bacterial multiplication in the host," she said. By not acting on essential genes in vitro, Escaich said antivirulence molecules should not induce selective pressure for resistant mutants.

Escaich added that although an antivirulence compound may not directly kill the bacteria in the host, "the compound makes the bacteria 


\section{TARGETS \& MECHANISMS}

more susceptible to innate immunity effectors that are circulating in the blood."

Furthermore, she said that bacteria treated with an antivirulence compound also become more sensitive to classical antibiotics, and thus combination therapy could be a potential application.

Mutabilis is developing antibacterial compounds against targets that are common to specific groups of pathogenic bacteria. The company's lead group of compounds inhibits resistance to cationic antimicrobial peptides (CAMP) in Gram-positive bacteria. These compounds are in the optimization phase to treat Staphylococcus and Streptococcus infections.

The company also has lipopolysaccharide (LPS) biosynthesis inhibitors for Gram-negative bacteria in early development.

"With the availability of a large number of sequenced bacterial genomes, it is now possible to select virulence targets that are conserved in several species," Escaich added.

Escaich did say the timing of expression of the virulence determinants during the infection process could be an issue. "An ideal target would have a constitutive expression" rather than an inducible one, she said.

According to Sperandio, although QseC expression is autoregulated and induced with epinephrine, the kinase is also constitutively expressed at basal levels in the bacteria.

Preclinical testing of LED209 in other bacterial pathogens is ongoing. The UT Southwestern researchers have filed a provisional patent application for LED209 and its derivatives. The application is available for licensing.

REFERENCES

1. Rasko, D. et al. Science; published online Aug. 22, 2008; doi:10.1126/science.1160354

Contact: Vanessa Sperandio, University of Texas Southwestern Medical Center, Dallas, Texas

e-mail: vanessa.sperandio@utsouthwestern.edu

2. Clarke, M.B. et al. Proc. Natl. Acad. Sci. USA 103, 10420-10425 (2006)

COMPANIES AND INSTITUTIONS MENTIONED

Achillion Pharmaceuticals Inc. (NASDAQ:ACHN), New Haven, Conn.

Mutabilis S.A., Paris, France

Paratek Pharmaceuticals Inc., Boston, Mass.

Tufts University, Medford, Mass.

University of Texas Southwestern Medical Center, Dallas, Texas 